\title{
La investigación bibliotecológica en América Latina: análisis de su desarrollo
}

\author{
Patricia Hernández Salazar *
}

Artículo recibido:

23 de mayo de 2005.

Artículo aceptado:

7 de julio de 2005.

\section{RESUMEN}

El objetivo del trabajo es presentar un panorama de la investigación bibliotecológica y de la ciencia de la información en algunos países de la región, en dos momentos diferentes: antecedentes (década de los ochenta) y situación actual (década del 2000). Para cada momento se identifican los objetivos, los temas o líneas de investigación, la problemática y las soluciones y con estos elementos se establecen los objetos de estudio. Se hace una comparación entre los antecedentes y la situación actual. Estos análisis nos permitieron determinar un posible paradigma disciplinario. Los países estudiados son Argentina, Brasil, Colombia y México.

* Centro Universitario de Investigaciones Bibliotecológicas de la UNAM, México. phs75599.servidor.unam.mx

INVESTIGACIÓN BiBLIOTECOLÓGICA, Vol. 20, Núm. 41, julio/diciembre, 2006, México, ISSN: 0187-358X. pp. 107-140 
Palabras clave: Investigación bibliotecológica; Investigación ciencias de la información; Paradigmas bibliotecológicos; Temas de investigación; Objetos de estudio.

\begin{abstract}
Library Science Research in Latin America: an analysis of its development

Patricia Hernández Salazar

The purpose of this paper is to give an overview of library and information sciences research in several countries of the region during two different moments in time: the lead-up of the 80's and today's situation (up to the year 2000). Objectives, research interests, problem areas and their solution are identified and compared for the two periods, to established study materials. These analyses allowed us to determine a possible disciplinary paradigm. The countries analysed are Argentina, Brazil, Colombia and Mexico.
\end{abstract}

Keywords: Library science research; Information science research; Library science paradigms; Lines of research.

\title{
INTRODUCCIÓN
}

$\mathrm{P}$ ara que una disciplina se considere consolidada debe tener un paradigma bien delimitado: un objeto de estudio perfectamente acotado; poseer marcos teóricos; determinar los caminos que se siguen para abordar los problemas y los fenómenos de investigación. Es decir, haber generado una estructura coherente de conceptos, supuestos teóricos y metodológicos para un campo de investigación específico.

La bibliotecología y la ciencia de la información son disciplinas que están en un estadio de búsqueda de consolidación tanto a nivel nacional como regional. En la región de América Latina existen varios países que realizan investigación en dichas disciplinas con el fin de generar las estructuras requeridas.

Entre esos países sobresalen Argentina, Brasil, Colombia y México, ya que la actividad de investigación se inició hace algunas décadas. De esto surgen 
varias preguntas ¿qué tan desarrollada está la disciplina en cada uno? ¿se tienen delimitados los objetos de estudio? ¿se siguen métodos y técnicas adecuadas para resolver los problemas de investigación? Para dar respuesta a estas interrogantes se realizó un análisis de la evolución de las disciplinas bibliotecología y ciencia de la información en cada país mencionado.

En la primera parte Surgimiento del interés por investigar en bibliotecología en América Latina se establecen los antecedentes de la investigación: el momento en el que se empieza a investigar; los temas o fenómenos de investigación; los problemas a los que se enfrentaron; y las soluciones que se les dieron a esos problemas.

Se hace un estudio exhaustivo de tal descripción con el fin de precisar el Panorama de los inicios, el cual conforma la segunda sección de este trabajo. Posteriormente, se presenta la Situación actual de la investigación en Argentina, Brasil, Colombia y México, seguida por los análisis cuantitativo y cualitativo de dicha situación, de los que resultó el apartado Panorama actual.

En la quinta división, Paradigmas, objetos de estudio y metodología se define el término paradigma y a partir de esta definición se determina el paradigma que se ha seguido en la bibliotecología. Por último se incluyen las Conclusiones y sugerencias que servirán de base para seguir estudiando tan interesante tema.

\section{SURGIMIENTO DEL INTERÉS POR INVESTIGAR EN Bibliotecología en AmÉrica LATINA}

Para tener una imagen lo más exacta posible sobre el desarrollo de las disciplinas bibliotecología y ciencia de la información se hace necesario precisar su evolución histórica, la cual puede rastrearse desde dos posibilidades: una, considerar que desde sus inicios la enseñanza de la bibliotecología ha seguido una tendencia basada en "formar para investigar"; es decir, que las instituciones de educación superior han formado a los alumnos en la búsqueda y solución de problemas; y otra, la creación de instituciones cuyo objetivo fuera justamente la generación de conocimiento nuevo.

Si retomamos la consideración de la enseñanza para investigar, los antecedentes se ubicarían en el surgimiento de la primera escuela de bibliotecología o ciencia de la información, lo cual sucedió en Argentina, donde la formación bibliotecológica a nivel superior empezó en 1922.

Algunos documentos que tocan el tema de los inicios de la investigación bibliotecológica coinciden en señalar la fundación de las escuelas de nivel superior como el antecedente primario; más aún, cuando se busca información 
sobre las líneas y/o proyectos de investigación, se pueden encontrar listados que describen los trabajos recepcionales o tesis que han desarrollado los alumnos egresados de tales escuelas para obtener sus títulos profesionales. Situación con la que no estoy totalmente de acuerdo, y que sostengo con los siguientes argumentos.

$\mathrm{Si}$ atendemos a los objetivos que en términos generales las instituciones de educación superior pretenden lograr en cada nivel educativo tenemos que, según la Universidad Nacional Autónoma de México (UNAM), en el nivel superior o licenciatura se pretende:

[...]dar al estudiante formación ética y cultural y capacitarlo científica y técnicamente dentro del campo de estudios correspondiente con el fin de que, como técnico, profesional, profesor o investigador pueda prestar servicios útiles a la sociedad. ${ }^{1}$

En cuanto a los estudios de maestría:

Tendrán al menos uno de los siguientes objetivos: iniciarlo en la investigación; formarlo para el ejercicio de la docencia de alto nivel; o desarrollar en él una alta capacidad para el ejercicio académico o profesional. ${ }^{2}$

Los estudios de doctorado tienen por objetivo:

[...]preparar al alumno para la realización de investigación original, así como proporcionarle una sólida formación disciplinaria, ya sea para el ejercicio académico o el profesional del más alto nivel. ${ }^{3}$

Si nos atenemos a estos propósitos, en sentido estricto, las tesis de doctorado serían los únicos trabajos que pueden considerarse como productos de conocimiento auténtico, es decir productos de una actividad real de investigación.

Cabe aquí delimitar el concepto que se tiene de investigación, la que se concibe como un proceso constante de generación de dudas y búsqueda de posibilidades para acallar esas dudas. Se investiga a partir de una necesidad de saber o conocer, de reconocer una carencia de conocimientos requerida para desarrollar un proceso cognoscitivo superior.

Dicha carencia debe ser planteada en términos de un problema o pregunta, debemos saber preguntar, si no existe pregunta es imposible concretar un problema, Bachelard afirmaba que: 
Ante todo es necesario saber plantear los problemas [...]. Es precisamente este sentido del problema el que indica el verdadero espíritu científico. Para un espíritu científico todo conocimiento es una respuesta a una pregunta. Si no hubo pregunta, no puede haber conocimiento científico... ${ }^{4}$

Ahora bien, encontrar soluciones a problemas que se plantean los seres humanos requiere de tres procesos intelectuales: pensar, conocer y razonar. Estos procesos tienen una relación cognoscitiva jerárquica, esto es, representan estadios que se van conteniendo uno en otro. Para razonar tendremos primero que pensar, y después conocer hasta llegar al tercer estadio que es precisamente razonar. Así, investigar no es un acto de búsqueda de respuestas en el cual se realiza una cadena de procesos intelectuales superiores de pensamiento, conocimiento y razonamiento.

Esta definición nos lleva a la otra posibilidad para establecer el antecedente más lejano en el campo de la investigación, es decir, a verla como la creación de instituciones planeadas para tal fin, situación que se consideró como la base para analizar el desarrollo de la investigación bibliotecológica. De acuerdo con esta eventualidad, Argentina también fue la pionera, puesto que en 1967 funda el Centro de Investigaciones Bibliotecológicas perteneciente a la Universidad de Buenos Aires.

Este Centro se creó con la finalidad de elaborar e implementar un Plan Nacional de Información y las investigaciones que se realizaron estuvieron relacionadas con el diagnóstico y la evaluación de la situación bibliotecaria en Argentina. ${ }^{5}$

A casi veinte años de su creación, en 1985, Josefa E. Sabor afirmaba:

La investigación en el campo bibliotecológico no parece haber tentado en demasía a los especialistas argentinos [...] Podemos decir que la investigación se halla entre nosotros casi en su etapa inicial, más aún, que en épocas pasadas fue más rica que en la actualidad. ${ }^{6}$

Entre las causas que propiciaban esta situación ella planteaba las siguientes:

- Deficiencia de las escuelas para enseñar técnicas de investigación.

- Inexistencia de un plan nacional o planes parciales de investigación.

4 G. Bachelard. La formación del espiritu científico. México: Siglo XXI, 1980. p. 16.

5 Susana Romanos de Tiratel. "La investigación bibliotecológica en Argentina", en La investigación bibliotecológica en la era de la información: Memoria del XXI Coloquio de Investigación Bibliotecológica y de la Información, 24-26 de septiembre de 2003. México: UNAM, Centro Universitario de Investigaciones Bibliotecológicas, 2004. p. 98.

6 Josefa E. Sabor. La investigación en bibliotecología. Buenos Aires: Asociación de Bibliotecarios Graduados de la República de Argentina, 1985. p.1. 
- Falta de fondos económicos para realizar investigaciones.

- Falta de organismos que apoyaran la investigación.

- Falta de estímulos para investigar.

- Carencia de información para desarrollar investigaciones.

En ese entonces la investigación giraba en torno a temas de aplicación práctica, tales como:

- Bases para estructurar servicios de información bibliográfica nacional.

- Naturaleza, estructura y estado actual de los servicios bibliotecarios.

- Mejoramiento de los procesos técnicos y los servicios bibliotecarios.

Como problemas detectaba:

- La falta de formulación de principios teóricos.

- Poco interés por cuestionar los principios tradicionales aceptados.

- La inexistencia de una metodología para abordar fenómenos bibliotecológicos.

- El profesional de la bibliotecología tenía las siguientes características:

- Carecía de aptitud y actitud positiva para investigar.

- Su formación estaba más cargada hacia aspectos técnicos.

- No sabía formular hipótesis.

- No cuestionaba los resultados que se presentaban.

- No poseía espíritu inquisitivo ni crítico. ${ }^{7}$

Josefina E. Sabor sugirió un plan para resolver esta problemática, en el que destacó que:

1. Las escuelas de bibliotecología [...] deben asumir la responsabilidad de impartir una enseñanza eficaz sobre metodología del estudio y la investigación [...].

2. Las escuelas de bibliotecarios incitarán a sus profesores a realizar trabajos de investigación, así como a dirigir a los alumnos en la iniciación de ese tipo de tareas[...].

3. Los centros, organismos o grupos de investigación deberán formular planes de trabajo definidos[...]. ${ }^{8}$

En orden cronológico, el siguiente país que crea un espacio específico para realizar labores de investigación en bibliotecología fue México. 
Todo se inicia en 1975 como un Proyecto de Investigación para la Dirección General de Bibliotecas, de la UNAM. La UNAM cuenta con un sistema de bibliotecas constituido por las bibliotecas de las escuelas de enseñanza media superior (bachillerato) profesional y de posgrado (escuelas y facultades), las de los centros e institutos de investigación y las de las dependencias administrativas. La Dirección General de Bibliotecas es la instancia encargada de controlar dicho sistema. En 1975 el sistema estaba integrado por 140 bibliotecas y según Rodríguez Gallardo este sistema presentaba los siguientes problemas:

- Poco desarrollo.

- La implantación de modelos bibliotecarios sin estudios previos de las necesidades de los usuarios.

- Carencia de estudios e investigaciones para desarrollar los servicios bibliotecarios.

- La necesidad de normalizar y optimizar los servicios bibliotecarios del sistema de bibliotecas de la UNAM.

- No se diseminaban experiencias y estudios en todo el país.

- Una falta de políticas de desarrollo de colecciones.

- La necesidad de incrementar sus usuarios y servicios.

- Problemas con el procesamiento técnico. ${ }^{9}$

Para solucionar estos problemas se crea el Proyecto de Investigación, cuyo objetivo fue:

[...] realizar aportes originales, principalmente en el campo de las bibliotecas universitarias y especializadas ya que son los dos tipos de biblioteca que forman el sistema bibliotecario de la UNAM. ${ }^{10}$

La idea fue contar con personal técnico que realizara investigación original y creativa en los diversos campos de la biblioteconomía. Los aspectos que se consideró necesario abordar fueron:

- Sistemas bibliotecarios.

- Sistemas de procesamiento técnico del libro: clasificación, catalogación y terminología.

9 Adolfo Rodríguez Gallardo. "La investigación bibliotecológica", en: Jornadas Mexicanas de Biblioteconomia (8: 1977: Guadalajara, Jal.). La problemática de las bibliotecas en México y sus soluciones: memorias. México: AMBAC, 1977. p.p. 30-32.

10 Adolfo Rodríguez Gallardo, op. cit., p. 32. 
- Nueva tecnología bibliotecaria.

- Recuperación de información bibliográfica por medios automatizados.

- Elaboración e implementación de servicios colectivos para la recuperación de información.

- Incremento, mantenimiento y actualización de colecciones universitarias.

- Necesidades de usuarios y uso de los diferentes tipos de material.

- Efectividad de los servicios bibliotecarios.

- Filosofía biblioteconómica de los usuarios.

Los resultados de esta primera incursión en investigación fueron tan satisfactorios que en 1981 se crea el Centro Universitario de Investigaciones Bibliotecológicas (CUIB) como una dependencia ubicada organizacionalmente dentro de la Coordinación de Humanidades, instancia que aglutina los centros e institutos que se dedican a la investigación de fenómenos sociales y humanísticos.

Para 1985 el interés por investigar aspectos bibliotecológicos respondió a dos situaciones básicas que Estela Morales enuncia así:

[...] a la urgencia de darle respuesta a un problema que afecta a una comunidad determinada, o a problemas que se plantea el propio investigador con el fin de profundizar sobre causas y consecuencias de un hecho, o de buscar el origen y comportamiento de una teoría sobre el usuario, la información, la organización, etc. $^{11}$

El propósito del Centro consistió en realizar investigaciones que ayudaran a la solución de problemas nacionales relacionados con las bibliotecas y centros de información del país, y en proporcionar apoyo a la educación, la investigación, la producción, la administración y la difusión de la cultura.

Las áreas de investigación que se abrieron entonces fueron:

- Historia de la bibliotecología.

- Educación bibliotecológica.

- Análisis bibliográfico.

- Usuarios.

- Evaluación: modelos matemáticos, informetría.

- Automatización.

- Tipos de biblioteca.

- Selección de materiales bibliográficos.

11 Estela Morales Campos. "Investigación Bibliotecológica”, en: Jornadas Mexicanas de Biblioteconomía (16: 1985: Pachuca, Hgo.). Memorias. México: AMBAC, 1986. p. 21. 
Para realizar sus funciones el Centro contó en ese entonces con 15 investigadores de tiempo completo.

Un análisis de los antecedentes de la investigación bibliotecológica en México nos lleva a determinar que evolucionó desde un estadio profesional y de administración (biblioteconomía) hasta uno disciplinario (bibliotecología), lo que se puede inferir fácilmente por los temas o líneas de investigación de cada época.

En 1977 se investigaba sobre administración de sistemas bibliotecarios, diseño de servicios para recuperar información y aspectos técnicos (clasificación, catalogación, desarrollo de colecciones), pero para 1985 aparecen nuevas líneas para desarrollar la disciplina, como son historia, educación, evaluación y usuarios.

En estos dos momentos de la investigación bibliotecológica se tuvieron como retos:

- Reclutar recursos humanos que estuvieran formados para investigar.

- Entrenar al personal en metodología de la investigación, estadística, automatización y tecnología educativa.

- Conformar una biblioteca especializada en bibliotecología.

- Conformar una colección sobre bibliotecología latinoamericana.

- Crear una base de datos que contuviera los registros de dicha colección.

Y los problemas eran:

- Salarios bajos.

- Carencia de presupuesto para formarse y actualizarse.

- Falta de equipos, laboratorios y bibliotecas adecuados.

- Mecanismos de difusión deficientes.

- Recortes presupuestales nacionales. ${ }^{12}$

La parte de la evolución histórica que sigue se sitúa en Colombia, donde en 1985 se crea el Centro de Investigaciones en Ciencias de la Información (CICINF), dependiente de la Escuela Interamericana de Bibliotecología, y según María Clemencia Molina:

[...] como parte fundamental para el desarrollo del currículo, ante la necesidad de crear situaciones académicas adecuadas a los cambios acelerados que exige la

12 Estela Morales Campos. "Las posibilidades de la investigación bibliotecológica en América Latina”, en Boletín de la Asociación Andaluza de Bibliotecarios. 4 (10): 22-24, enero-marzo, 1988. 
profesión y al vertiginoso avance de conocimientos y tecnologías en el campo de la información. ${ }^{13}$

Los objetivos del Centro fueron:

- Realizar investigaciones para acrecentar el conocimiento en el área, tanto en Colombia como en América Latina.

- Contribuir al enriquecimiento de la literatura profesional.

- Fomentar la actividad investigativa.

Como líneas de investigación se planteaban:

- Naturaleza de la información y aspectos sociopsicológicos de las relaciones entre generadores y usuarios de la información.

- Teoría y estructura de la información desde una perspectiva de medición (bibliométrica y cienciométrica).

- Epistemología de la bibliotecología y de la ciencia de la información.

- Análisis y evaluación de los procesos históricos.

- El profesional para el trabajo en información: formación, status y mercado de trabajo.

- Diseño, operatividad y evaluación de unidades y servicios de información.

- Promoción de la lectura. ${ }^{14}$

En un estudio realizado en la década de los noventa, Rocío Herrera ${ }^{15}$ presentó como problemas de la investigación en ese país:

- Un porcentaje muy elevado de trabajos de tipo exploratorio-descriptivo.

- La debilidad del cuerpo teórico, la base era conocimiento empírico generado por ensayo-error.

- Se percibía a la investigación como una actividad marginal a la profesión.

- Los profesionales recibían una formación repetitiva, mnemotécnica, y tendiente al desempeño de un oficio.

- Los resultados no eran acumulativos.

- No se habían aprobado tesis.

13 María Clemencia Molina Escobar. "Desarrollo y perspectiva de la investigación en bibliotecología y archivología en Colombia”, en: Encuentro de Educadores e Investigadores de Bibliotecología, Archivología y Ciencia de la Información de Iberoamérica y el Caribe (4: 1998: Maracaibo, Venezuela). Venezuela: [s.e.], 1998. h. 9.

14 María Clemencia Molina Escobar. op. cit., h. 9-10.

15 Rocío Herrera Cortés. "Panorama de educación e investigación de la Bibliotecología y Ciencias de la Información en Colombia”, en Ramírez Leyva, Elsa. Reunión de Investigadores y Educadores de Iberoamérica y del Caribe en el Área de Bibliotecología y Ciencia de la Información. México: UNAM, Centro Universitario de Investigaciones Bibliotecológicas, 1996. pp. 259-261. 
- Existía una gran tradición empírica que destacaba aspectos prácticos del ejercicio profesional.

- Faltaba formación analítica, evaluativa y de seguimiento.

- No se tenía una definición nacional de una necesidad de desarrollo científico y tecnológico.

- Había aislamiento entre los investigadores y las instituciones que se dedicaban a la investigación, tanto a nivel nacional como regional.

- Faltaba la retroalimentación, la réplica de investigaciones y la confrontación de resultados.

- Había poca producción de literatura escasa y ésta era mal difundida.

- No existían grupos dedicados de tiempo completo o al menos de forma parcial a la investigación.

Frente a lo anterior se propusieron las siguientes perspectivas constructivas:

- Formar investigadores.

- Trabajar entre pares.

- Generar programas de investigación al interior de las universidades.

- Desarrollar proyectos en conjunto con otras universidades.

- Crear la profesión de investigador.

- Difundir los resultados de las investigaciones.

- Capacitar administradores de investigación.

- Utilizar las nuevas tecnologías para la implantación de políticas.

Conviene hacer notar que desde sus inicios el CICINF fue concebido como una instancia de desarrollo para la disciplina, por lo que su problemática en esos momentos era muy amplia.

Un país que se considera importante dentro del desarrollo de la investigación bibliotecológica en América Latina es Brasil, en este país no existen instancias específicas para realizar investigación, sin embargo es pertinente incluirlo en este análisis. Como sustento de esta consideración está el hecho de que sus fuentes de investigación son mayoritariamente los cursos de posgrado, principalmente el doctorado.

Se puede afirmar que Brasil fue pionero en el establecimiento de estudios de doctorado, pues desde 1980 aparece el Doctorado en Comunicación con tendencia hacia el área de bibliotecología, dependiente de la Escuela de Comunicación de la Universidad Federal de Río de Janeiro. En esta misma universidad existen planes de maestría y doctorado en ciencias de la información, la primera desde 1975 y el segundo aparece en 1992. Este doctorado se ha trasladado a la Universidad Federal Fulminense. 
La Universidad de Sao Paulo tiene desde 1980 un doctorado en ciencias de la comunicación y artes, con una línea de investigación en bibliotecología. Asimismo en la Universidad de Brasilia cuentan con programas de maestría (1978) y doctorado (1992). Estos programas de doctorado han producido tesis que pueden considerarse trabajos que reflejan una producción de conocimiento auténtico.

Para 1983, el Consejo Nacional de Desarrollo en Ciencia y Tecnología de ese país, había realizado tres estudios (1978, 1980 y 1983) sobre la investigación en bibliotecología, los cuales arrojaron los siguientes resultados:

- Los principales productos que representaban la investigación eran tesis de maestría y doctorado.

- La investigación era desarrollada por docentes y alumnos de posgrado.

- Los temas que se abordaron en ese periodo (1978-1983) fueron:

- Estudios de usuarios.

• Enseñanza.

- Lenguajes de indización.

- Sistemas bibliotecarios.

- Transferencia de información.

- Evaluación de colecciones.

- Problemas especiales de bibliotecas públicas.

- Se trabajaba en forma individual.

- Se usaban métodos cuantitativos.

- Las técnicas de investigación empleadas eran:

- Entrevistas.

- Cuestionarios.

- Observación.

- Análisis de documentos.

- Bibliometría. ${ }^{16}$

Los obstáculos que se percibieron fueron los siguientes:

- Falta de recursos humanos adecuados, tanto cuantitativa como cualitativamente.

- Falta de disponibilidad de recursos bibliográficos.

- Carencia de conocimientos sobre métodos de investigación.

- Carga excesiva de horas clase y de actividades administrativas.

16 Nice Figueiredo. Research in library and information sciences in Brazil, Seminario pre-conferencia IFLA sobre educación para la investigación e investigación para la educación. Bogotá: ALEBCI, 1983. pp. 72-78. 
- No existía tradición en investigación.

- Las teorías existentes eran incipientes y no muy diferenciadas.

- No existía una infraestructura adecuada en cuanto a colecciones, espacios y equipo.

- Se carecía de un financiamiento adecuado para investigar.

- No había incentivos institucionales para asistir a eventos de difusión.

- Había aislamiento intelectual.

- La comunicación entre profesores era deficiente.

Como posibles soluciones se planteó:

- Formar investigadores.

- Retomar teorías existentes en otras áreas.

- Utilizar métodos adecuados.

\section{Panorama de los inicios}

Con el fin de tener una primera aproximación al escenario que prevalecía en el inicio de la actividad de investigación en los países estudiados, se hicieron dos análisis, uno cuantitativo y otro cualitativo. El cuantitativo describe los temas de investigación, los problemas que se presentaban y sus posibles soluciones.

En cuanto a los temas, en el Cuadro 1. Temas de investigación en la década de los ochenta (se anexan todos los cuadros al final) se percibe que se abordaron 17 temas en 25 proyectos. El país que menos temas desarrolló fue Argentina, con tres $(12 \%)$; los 22 temas restantes se distribuyeron equitativamente entre los tres países: México, ocho (32\%), Colombia, siete (28\%) y Brasil, siete (28\%).

México, Colombia y Brasil coincidieron en los temas de educación, usuarios y evaluación; Argentina y México en procesos técnicos; México y Colombia en historia de la bibliotecología. Es decir que sólo cinco temas (20\%) fueron de interés regional, y los 20 restantes despertaron interés sólo a nivel nacional. Si cerramos los temas en grandes subcampos disciplinarios, las investigaciones se ubican de la siguiente manera:

Organización: 12

- Bibliografía nacional/bases-Argentina.

- Servicios bibliotecarios-Argentina.

- Procesos técnicos/análisis bibliográficos-Argentina y México.

- Evaluación: colecciones, servicios, unidades, diseño y operatividadMéxico, Colombia y Brasil. 
- Selección-México.

- Tipos de bibliotecas-México.

- Lenguajes de indización-Brasil.

- Sistemas bibliotecarios-Brasil.

- Bibliotecas públicas-Brasil.

Usuarios: 3-México, Colombia y Brasil.

Educación: 3-México, Colombia y Brasil.

Fundamentos teóricos: 2 - Colombia.

- Teoría y estructura de la información.

- Epistemología de la bibliotecología y de la ciencia de la información, Historia: 2-México y Colombia.

Tecnologías: 2

- Automatización-México.

- Transferencia de información-Brasil.

Lectura: 1 - Colombia.

Como se puede observar casi la mitad (48\%) son temas relacionados con el fenómeno de la organización; en orden decreciente aparecen la educación y los usuarios con $12 \%$ cada tema ( $24 \%$ ); a éstos los siguen fundamentos teóricos, historia y tecnologías con $8 \%$ cada uno (24\%); y por último aparece la lectura con un $4 \%$.

Con respecto a la problemática para realizar investigaciones en estos países se detectó que existían 16 problemas (ver Cuadro 2. Problemas en la investigación bibliotecológica: antecedentes). Los cuatro países coinciden en señalar como un problema la falta de recursos con aptitudes y actitudes para investigar; tres de ellos, Argentina, Colombia y Brasil apuntan la falta de principios teóricos.

Seis problemas se presentan en dos países, con diferentes coincidencias: en Argentina y Brasil, uno; en México y Brasil, dos; en México y Colombia, uno; y en Colombia y Brasil, dos. De acuerdo con esto, se puede inferir que el $50 \%$ (8) de los problemas son comunes a dos, tres y cuatro países.

Si integráramos los problemas en grandes apartados tendríamos la siguiente distribución:

Aislamiento de investigadores (problemas 10,

$11,12$ y 16$)$.

Falta de recursos y de aptitudes y actitudes para investigar

(problemas 2, 4, 14 y 15).

Falta de principios teóricos y metodológicos

(problemas 1,3 y 9 ).

Mecanismos deficientes sobre resultados de difusión 
Presupuestos inadecuados (problemas 5 y 6 ).

Infraestructura deficiente (problema 7).

En el cuadro 3. Soluciones a problemas: antecedentes, aparecen las alternativas que cada país consideró convenientes para paliar la problemática detectada, el total de soluciones asciende a 13.

Como podremos recordar existían ocho problemas que afectaban a más de un país, sin embargo sólo una alternativa (formar al personal en metodología de la investigación, estadística, automatización y tecnología educativa) fue considerada por los cuatro países. Argentina también apuntó como problema que los recursos humanos no tenían aptitudes y actitudes para investigar, sólo que ellos buscaron la solución mediante la formación "eficaz" sobre metodología de la investigación en las escuelas.

Relacionamos los problemas con las soluciones con el fin de detectar si hubo coincidencia en la forma para resolver los problemas en los países; los problemas se enuncian de acuerdo con los grandes apartados anotados en párrafos anteriores:

\begin{tabular}{|c|c|}
\hline Problemas & Soluciones \\
\hline Aislamiento de investigadores (cuatro incidencias) & $\begin{array}{l}\text { - Desarrollar proyectos colectivos - Colombia } \\
\text { - Trabajar entre pares - Colombia }\end{array}$ \\
\hline $\begin{array}{l}\text { Falta de recursos con aptitudes y actitudes para } \\
\text { investigar (cuatro) }\end{array}$ & $\begin{array}{l}\text { - Formación sobre metodología por parte de las } \\
\text { escuelas - Argentina } \\
\text { · Formar al personal en metodología, estadística, } \\
\text { automatización y tecnología educativa - México, } \\
\text { Colombia y Brasil } \\
\text { - Motivar a los docentes a realizar trabajos de } \\
\text { investigación - Argentina } \\
\text { - Crear la formación de investigador - Colombia }\end{array}$ \\
\hline Falta de principios teóricos y metodológicos (tres) & $\begin{array}{l}\text { - Retomar teorías de otras disciplinas - Brasil } \\
\text { - Utilizar métodos adecuados - Brasil }\end{array}$ \\
\hline $\begin{array}{l}\text { Mecanismos de difusión de resultados } \\
\text { deficientes (dos) }\end{array}$ & - Difundir los resultados - Colombia \\
\hline Presupuestos inadecuados (dos) & $\begin{array}{l}\text { - Formular planes de trabajo definidos - Argentina } \\
\text { - Capacitar administradores de } \\
\text { investigación - Colombia }\end{array}$ \\
\hline Infraestructura inadecuada (una) & $\begin{array}{l}\text { - Conformar colecciones sobre bibliotecología } \\
\text { latinoamericana / México } \\
\text { - Crear bibliotecas especializadas en } \\
\text { bibliotecología / México }\end{array}$ \\
\hline
\end{tabular}

Como se puede apreciar se le dio solución a la totalidad de los problemas y en la mayoría de los casos fue más de una. 
La siguiente etapa consistió en hacer un análisis cualitativo, el cual nos permitió llegar a las siguientes conclusiones:

- La necesidad de investigar surgió como un interés por resolver problemas profesionales y no para desarrollar la disciplina.

- En ese momento no se establecieron objetos de estudio, sin embargo, del análisis cuantitativo de los temas de investigación presentado, se deriva que el objeto de estudio (desde una perspectiva paradigmática) fue la organización. Las líneas o temas de investigación que se siguieron estuvieron circunscritas básicamente a aspectos técnico/profesionales de organización y servicios.

- Solo en Colombia se abordó el tema sobre fundamentos teóricos.

- Argentina estudiaba únicamente temas relacionados con la organización.

- Tanto México como Brasil trabajaron más sobre organización.

- Un problema que apareció como constante en los cuatro países fue que las personas que se dedicaban a la investigación no estaban formadas para investigar. La alternativa para resolver esto fue expresada por los cuatro países que lo declararon, la propuesta fue formar a su personal sobre metodología de la investigación, ya sea en las propias instancias de investigación o en las instituciones educativas.

- En esa época hubo poco interés por abordar fenómenos relacionados con los fundamentos teóricos y metodológicos, lo que se reflejó en la problemática. Si bien Argentina, Colombia y Brasil declararon esto como problema, Argentina no presentó ninguna solución.

- Otro obstáculo fue el aislamiento de los investigadores, las soluciones que se propusieron desarrollar proyectos colectivos y trabajar entre pares, lo que redundaría en la creación y/o consolidación de comunidades epistémicas.

- Si bien México y Colombia detectaron que la publicación de resultados era escasa y los canales de difusión y distribución eran deficientes, sólo Colombia intentó resolver esa situación.

- Resalta el hecho de que sólo para México fuera importante crear bibliotecas especializadas en bibliotecología y conformar colecciones sobre la producción de los especialistas en bibliotecología de América Latina.

\section{SitUACiÓN ACTUAL}

¿Qué ha pasado con la investigación en bibliotecología y ciencia de la información desde entonces hasta ahora, inicio del siglo XXI? 


\section{Argentina}

En 1996 el Centro de Investigaciones Bibliotecológicas pasa a ser Instituto de Investigaciones Bibliotecológicas y queda adscrito a la Facultad de Filosofía y Letras de la Universidad de Buenos Aires.

Los objetivos actuales del Instituto son:

* Elaborar y desarrollar proyectos de investigación, de transferencia o de extensión;

* Formar investigadores;

* Asesorar a diferentes entidades;

* Contribuir al perfeccionamiento y especialización de los docentes;

* Desarrollar cursos de especialización, seminarios, etcétera.

* Fomentar la producción y distribución de publicaciones, etc. ${ }^{17}$

Si recordamos, el objetivo original del Centro fue implementar un Plan Nacional de Información, es decir, buscaba apoyar la profesión, en la actualidad refleja una tendencia hacia el desarrollo de la disciplina y ya se percibe una intención de generar teorías y formar en aspectos relacionados con la investigación.

Los fenómenos que actualmente se están estudiando son :

- Procesos de búsqueda de información y modalidades de intervención.

- Automatización de las bibliotecas universitarias argentinas frente al nuevo milenio.

- Indicadores de la actividad de investigación aplicados a las revistas argentinas.

- Bibliotecas, sociedad de la información y tecnologías: una perspectiva desde la automatización y los servicios de las bibliotecas de acceso público.

- Historia del libro, de las bibliotecas y de la lectura: periodo colonial, independiente y siglo XX.

- Orígenes de la biblioteca pública.

- Indicadores de visibilidad de la producción en economía, historia y sociología.

- Control bibliográfico en ciencias humanas.

El personal que realiza las investigaciones son profesores con dedicación exclusiva y semi-exclusiva, y los auxiliares docentes bajo la supervisión de un profesor/investigador, su número aproximado es de ocho. 
Problemática:

- Preeminencia de la investigación aplicada.

- Ausencia de investigaciones teóricas.

- La mayoría de profesionales ignoran los métodos de indagación científica en la recopilación de datos.

- La construcción del saber se hace a partir de la práctica, la declaración autorizada y la intuición.

- Existen barreras de comunicación entre profesionales e investigadores.

- Los profesionales no aplican los hallazgos que resultan de la investigación para la solución de sus problemas.

- Sistemas de difusión deficientes o en algunos casos inexistentes.

- Falta de formación para investigar.

- Baja producción de literatura bibliotecológica argentina.

Existe un gran optimismo hacia el futuro ya que a partir de la década de los noventa, la investigación bibliotecológica en las universidades se ha formalizado e institucionalizado. Se considera que existe un notable crecimiento de la disciplina puesto que se han obtenido los siguientes logros: la creación de una revista especializada a nivel académico; el sostenimiento de una serie monográfica; el mejoramiento de los servicios de información para los investigadores y el fortalecimiento de las relaciones intrainstitucionales.

\section{México}

El objetivo actual del Centro Universitario de Investigaciones Bibliotecológicas es:

[...] contribuir al enriquecimiento del cuerpo de conocimientos de la disciplina y mantener una vinculación y retroalimentación permanente con la sociedad a través de la docencia, de actividades de difusión, divulgación y medios de diseminación de resultados y productos, mismos que están dirigidos a diferentes sectores de la sociedad. ${ }^{18}$

Para cubrir este objetivo la investigación está organizada en cinco áreas y 19 líneas, a saber:

Área I. Fundamentos de las ciencias bibliotecológica y de la información: Líneas de investigación:

18 Centro Universitario de Investigaciones Bibliotecológicas. México: UNAM, Centro Universitario de Investigaciones Bibliotecológicas, [s.f.]. p. 2. 
- Fundamentación epistemológica.

- Fundamentación ética.

- Fundamentación histórico-social.

Área II. Información y sociedad:

Líneas de investigación:

- Educación para informar.

- Estudios de comunidad y grupos sociales.

- Historia de la bibliotecología.

- Lectura.

- Políticas y legislación de la información.

Área III. Sistemas de información:

Líneas de investigación:

- Desarrollo de colecciones.

- Fuentes de información.

- Metría de la información.

- Usuarios de la información.

Área IV. Análisis y sistematización de la información documental:

Líneas de investigación:

- Lenguaje de búsqueda de información.

- Normalización y control bibliográfico.

- Sistemas de clasificación.

Área V. Tecnologías de la información:

Líneas de investigación:

- Evolución de las tecnologías de la información.

- Interfases para la transferencia y acceso a la información digital.

- Marco conceptual de las tecnologías de la información.

- Recursos de información basados en tecnologías.

El trabajo de investigación lo realizan 24 investigadores de tiempo exclusivo: catorce con grado de doctor, ocho con maestría y dos con licenciatura.

Problemática:

- Reclutar personal formado.

- Difusión ineficiente de las publicaciones producidas.

- Los profesionales no aplican los resultados de las investigaciones.

- Escasa generación de marcos teóricos. 
- Falta de replicabilidad de los resultados.

- Uso de métodos cuantitativos en la mayoría de investigaciones.

- El trabajo se realiza en forma individual. ${ }^{19}$

\section{Colombia}

El CICINF persigue los siguientes objetivos:

- Contribuir al progreso de la nación con programas de investigación innovativos.

- Incrementar la actividad de investigación entre los profesores, egresados y estudiantes.

- Solucionar problemas en el diseño y operación de sistemas y servicios de información. ${ }^{20}$

Sus líneas de investigación son:

- Contextualización de la información.

- Caracterización de los sistemas de información

- Organización de la información

- Tecnologías de la información

- Gerencia de los sistemas de información

Se apuntan como retos más que como problemas, y los siguientes aspectos:

- Generar un campo teórico sólido y aceptado por las comunidades científicas.

- Consolidar el trabajo interdisciplinario y en grupo.

- Lograr que los investigadores traduzcan conocimiento auténtico y por consiguiente publicaciones que difundan los hallazgos.

- Conformar una tradición de investigación reconocida.

- Crear conciencia sobre la importancia de la investigación para el desarrollo y reconocimiento de la profesión, tanto en egresados como en empleadores. $^{21}$

19 Informe de actividades agosto 2003-agosto 2004. México: Universidad Nacional Autónoma de México, 2004. p. 15.

20 Edilma Naranjo Vélez y Rendón Giraldo Nora Elena, "La investigación bibliotecológica y las comunidades científicas en un contexto colombiano", en La investigación bibliotecológica en la era de la información: memoria del XXI Coloquio de Investigación Bibliotecológica y de la Información, 24-26 de septiembre de 2003. México: UNAM, Centro Universitario de Investigaciones Bibliotecológicas, 2004. p. 81. 


\section{Brasil}

Precisar con exactitud las características actuales de la investigación bibliotecológica en este país fue imposible. No se encontró alguna publicación que tratara sobre esto, ya que la investigación está dispersa en tantas instituciones educativas como programas de posgrado existen. Se consultaron sitios de las instituciones que tenían los doctorados y desafortunadamente sólo mencionan que existe posgrado, sin proveer información sobre planes de estudio, líneas o proyectos de investigación; asimismo, en las opciones de investigación no se desglosan las líneas de investigación ni los proyectos.

Se recuperó un documento que analiza los trabajos presentados en las reuniones de la Asociación Nacional de Investigación y Posgrado en Ciencias de la Información en ese país, entre 1994 y 2000, los resultados que presenta son:

Temas de investigación:

- Información tecnológica.

- Representación del conocimiento/indización.

- Teoría de la clasificación.

- Nuevas tecnologías/bases de datos/fuentes de información y la educación a distancia.

- Información y sociedad/acción cultural.

- Comunicación científica.

- Formación profesional/mercado de trabajo.

- Planeación de sistemas/inteligencia competitiva.

- Epistemología de la ciencia de la información. ${ }^{22}$

Los tipos de trabajo que se consideraron investigaciones fueron: disertaciones de maestría, investigaciones individuales e investigaciones en grupo, así como monografías escolares.

Y como problemas plantea:

- Que hay deficiencia en el uso de métodos y técnicas de investigación.

- Que la mayoría de los trabajos son producto de investigadores en formación (estudiantes de maestría).

22 Suzana P. M Mueller; Miranda Antonio L.C. y Emir J. Suaiden, A pesquisa em Ciencia da informacao no Brasil: Analise dos trabalbos apresentados no IV Enancib, Brasilia 2000, Documento impreso en computadora. h 15. 
Al igual que en el apartado sobre los inicios, para establecer la situación que prevalece en estos tiempos se hicieron dos análisis, uno cuantitativo y otro cualitativo.

El análisis cuantitativo nos arrojó los siguientes resultados. Los temas ascendieron a 23 y se presentan en el Cuadro 4. Temas de investigación en la década del 2000, en el cual se percibe que un tema podía tener dos o tres fenómenos de investigación o que algunos países coincidieron en éstos, por lo que el número total de investigaciones fue de 40 . El país que está realizando mayor número de proyectos es México, con 19 (47.5\%), y el que hace menos es Colombia con 4 (10\%).

Sobresalen los temas que en un mismo país tienen más de un proyecto como son:

Argentina: Automatización $\quad 2$

México: Tecnologías de Información 4

Fundamentación 3

Usuarios 2

Esto refleja una profundización sobre ciertas líneas de investigación.

En nueve temas coinciden dos países:

Búsqueda-Argentina y México.

Historia-Argentina y México.

Control bibliográfico-Argentina y México.

Fundamentación-México y Brasil.

Educación-México y Brasil.

Usuarios de la información-México y Brasil.

Sistemas de clasificación- México y Brasil

Tecnologías de información-México y Colombia.

Sistemas de información-Colombia y Brasil.

El resto de los temas interesan sólo a un país. Si cerramos nuevamente los temas en grandes subcampos, tenemos lo siguiente:

Organización: 18 temas

- Búsqueda: procesos, lenguaje - Argentina y México.

- Indicadores: actividad de investigación, producción en economía, historia y sociología-Argentina (2). 
- Bibliotecas públicas-Argentina.

- Control bibliográfico en ciencias humanas y normalización-Argentina y México.

- Políticas y legislación de la información-México.

- Desarrollo de colecciones-México.

- Metría de la información-México.

- Fuentes de información-México.

- Sistemas de clasificación-México y Brasil.

- Sistemas de información: caracterización, teoría, planeación/inteligencia competitiva, gerencia-Colombia y Brasil.

- Organización-Colombia.

- Comunicación científica-Brasil.

- Representación del conocimiento/indización-Brasil.

Tecnologías: 8

- Automatización: bibliotecas universitarias, impacto-Argentina (2).

- Tecnologías de información: marco conceptual, evolución, recursos de información-México (4) y Colombia.

- Información tecnológica-Brasil.

Usuarios: 4

- Estudios e información y sociedad-México (2) y Brasil.

- Contextualización de la información-Colombia.

Fundamentación: 4

- Epistemológica, ética e histórico social-México (3) y Brasil.

Educación: 3

- Formación profesional/mercado de trabajo-México y Brasil.

- Educación a distancia y tecnologías-Brasil.

Historia: 2-Argentina y México

Lectura: 1 - México.

Los datos expuestos muestran que casi la mitad de los proyectos, 45\%, corresponde al área de organización, los temas que le siguen son tecnologías con $20 \%$; usuarios con $10 \%$; fundamentación con $10 \%$; educación con 7.5 $\%$; historia con el $5 \%$; y lectura con un $2.5 \%$. 
En lo que se refiere a los problemas que se detectan actualmente, en el Cuadro 5. Problemas en la investigación bibliotecológica: década del 2000 se aprecia que el número de problemas asciende a 19. Ocho de ellos son comunes a dos países: Argentina y Brasil dos; Argentina y cuatro; y México y Colombia dos; de lo que se deduce que un poco más de la mitad de los problemas $52.6 \%$ son privativos de cada país.

Nuevamente integramos los problemas en grandes rubros con el fin de percibir su comportamiento, lo que arrojó los siguientes resultados:

Falta de formación para investigar (problemas 3, 4, 9, 10, 16 y 18$)$.

Falta de aplicación y replicabilidad de los resultados (problemas 5, 6, 12, 15 y 17).

Escasa generación de marcos teóricos y conocimiento auténtico (problemas 1, 2, 13, 14 y 19).

Mecanismos de difusión deficientes (problemas 7 y 8). 2

Trabajo individual (problema 11).

De estos resultados se infiere que existen tres grandes problemas que representan la mayoría (84.2\%): falta de formación en investigación (31.6\%); falta de aplicación de resultados (26.3\%); y escasa generación de marcos teó$\operatorname{ricos}(26.3 \%)$.

En los documentos revisados no se encontraron las soluciones que pudieran resolver las dificultades planteadas, lo cual puede deberse a que estamos en la etapa de identificación de la problemática.

Se analizaron cualitativamente los resultados numéricos, análisis que nos proporcionó el estado que actualmente presenta la investigación bibliotecológica y de la ciencia de la información. Incluimos a continuación sus rasgos principales:

- Los objetivos de las instancias que hacen investigación en América Latina están dirigidos a desarrollar la disciplina, con una marcada vinculación social. Esta vinculación se busca de tres maneras: resolviendo problemas que afecten a la sociedad; divulgando los resultados; y formando futuros investigadores. Asimismo, se advierte la intención de producir medios de diseminación para los resultados, con esto se entiende que al hacer investigación no sólo se resuelven problemas sociales y disciplinarios sino que tienen que quedar registros para que los hallazgos puedan retomarse, replicarse y discutirse.

- No se establecen cabalmente los objetos de estudio, ni análisis de los temas y proyectos de investigación, pero podemos afirmar que sigue 
prevaleciendo la organización, aunque han aumentado los temas sobre tecnologías, usuarios y fundamentación. Estos datos muestran una contradicción con los objetivos en lo que se refiere al desarrollo de la disciplina, como todos sabemos la consolidación de un campo de conocimientos requiere generar marcos teóricos, y en esta área estamos trabajando muy poco.

- Lo anterior se ve reforzado por el hecho de que entre los problemas sin resolver está precisamente la escasa generación de propuestas teóricas y metodológicas, y de que cuando éstas existen son poco sólidas y aceptadas.

- Un reto importante sigue siendo el hecho que las plantas de investigadores o profesores/investigadores no estén formadas para realizar actividades de producción de conocimiento auténtico; es decir, que sepan de epistemología o teoría del conocimiento (¿qué es?, ¿cómo puede generarse?, ¿cómo se genera un concepto?, ¿cómo se establecen las relaciones entre conceptos?) y de metodología (¿cómo se delimita un problema de investigación?; ¿cómo se delimita el fenómeno de estudio?, ¿cuál es el mejor camino para resolver ese problema? y ¿cómo registrar los resultados?) tanto cualitativa como cuantitativa. $\mathrm{Si}$ nos detenemos a reflexionar sobre esto nos daremos cuenta que existe una relación casi simbiótica entre la necesidad de cuadros formados para investigar y la escasa o nula generación de fundamentos teóricos.

- Un problema reiterado es la falta de vinculación entre profesionales e investigadores, lo que se refleja en la escasa o nula aplicación de los resultados de la investigación en contextos profesionales.

- No se encontraron soluciones a los problemas planteados.

Un análisis comparativo entre la situación de los inicios y la actual, arroja las siguientes conclusiones:

- El objetivo de la investigación en bibliotecología y ciencia de la información ha evolucionado: en los ochenta fue resolver problemas profesionales, en la actualidad se pretende desarrollar la disciplina.

- En ambos momentos no existe un objeto de estudio delimitado, se infiere que éste es la organización. Pero actualmente han aumentado los proyectos sobre Fundamentación (México) y uso de la información/ usuarios (México, Colombia y Brasil).

- Hubo un incremento significativo, del $60 \%$, en el número de proyectos, en los inicios se realizaron 25 y actualmente se están desarrollando 40 . 
- Un problema que no se ha resuelto es la deficiente formación de los investigadores en aspectos metodológicos.

- Los resultados de las investigaciones no se aplican ni replican, lo que ha provocado que no hayamos generado marcos teóricos y metodológicos consistentes, por lo que no existe una verdadera comunidad epistémica, tanto a nivel nacional como regional.

- Prevalecen las dificultades relacionadas con la escasa producción, y la que existe no es difundida adecuadamente.

- Parece haberse resuelto los problemas de financiamiento y falta de infraestructura, puesto que no se reiteran en el panorama de la situación actual.

- En la época actual se detectó un problema que cabe resaltar, la falta de relación entre las comunidades de profesionales y las de investigadores, lo que limita la consolidación de las disciplinas de bibliotecología e información.

\section{PARAdigmas, obJetos DE ESTUdio y METODOlOgía}

Surge la pregunta ¿cómo podemos generar o consolidar nuestros marcos teóricos? Debemos delimitar nuestro campo de conocimientos, sus límites, sus objetos y sus fenómenos de estudio, las relaciones entre esos objetos y los fenómenos, las formas de abordarlos (métodos, técnicas e instrumentos); concretamente, debemos precisar el paradigma que guía nuestro quehacer investigativo.

Partamos del principio ¿qué significa el término paradigma? Según Ku$\mathrm{hn},{ }^{23}$ un paradigma es una estructura coherente constituida por una red de conceptos, creencias teóricas y metodológicas entrelazadas que permiten la selección, evaluación y crítica de temas, problemas y métodos, y que es legítima para un campo de investigación.

Esta estructura coherente crea o debe crear una red de compromisos entre los miembros de una comunidad científica o epistémica. Para Kuhn, la ciencia se desarrolla dentro de un paradigma, en el cual se van acumulando los conocimientos; es decir, que lo concibe como el principio rector del conocimiento y de la existencia humana.

De acuerdo con esta definición podríamos preguntarnos ¿tenemos un paradigma bibliotecológico? A continuación trataremos de responder a esta interrogante. 
Un paradigma debe delimitar el objeto de estudio de la disciplina, entendiéndolo (al objeto de estudio) como la forma o el término del acto del conocimiento; el objeto es la finalidad, el propósito, lo que se elige. Tenemos así que el objeto de estudio es el propósito o término de estudio de un campo de conocimientos, y de aquí nos surge otra pregunta ¿cuál es el objeto de estudio de la bibliotecología y el de la ciencia de la información?

Empiezan aquí los problemas de delimitación del objeto de estudio, cuando en nuestros planes de estudio y/o manejo del lenguaje común hablamos de bibliotecología como sinónimo de ciencia de la información, o cuando los unimos y generamos frases como bibliotecología y estudios o ciencia de la información.

Para tratar de precisar el objeto de estudio de cada disciplina tendríamos que hacer un estudio de introspección para ubicar el término, forma o propósito que nos sirva de base para desarrollar nuestras actividades de investigación.

A manera de ejemplo revisemos lo siguiente. Brasil, de acuerdo con su Consejo Nacional de Desarrollo en Ciencia y Tecnología (CNPQ), apoyado en las orientaciones de la UNESCO, define que la ciencia de la información:

[...] designa un campo más amplio, de propósitos investigativos y analíticos, interdisciplinario por naturaleza, que tiene por objetivo el estudio de los fenómenos ligados a la producción, organización, difusión y utilización de la información en todos los campos del saber. ${ }^{24}$

De esta definición se deriva que su objeto de estudio son los procesos de producción, organización, difusión y utilización de la información, es decir la información y sus procesos.

Para México, el objeto de estudio es la relación entre la información, entendida como el registro y flujo del conocimiento y el uso/usuario, entendido como el sujeto que requiere y promueve la circulación social de los medios que contienen la información.

Si analizamos estos dos objetos de estudio nos daremos cuenta que si bien se parecen, le conceden un propósito diferente a cada disciplina; la ciencia de la información estudia a la información, y la bibliotecología, la relación información/ usuario, por lo que sus fenómenos de investigación pueden variar en grado de importancia y en la manera de abordarlos. Cuando fusionamos estas dos disciplina y hablamos de la bibliotecología y ciencia de la información como si fuesen una tendríamos que delimitar cuál de los dos objetos de estudio prevalecerá.

24 Marlene de Oliveira, "La investigación científica en ciencia de la información: análisis de la investigación financiada por el CNPQ (Brasil) en el período de 1984 a 1993”, en Investigación bibliotecológica: archivonomía, bibliotecología e información. 17 (34): p. 71. Enero-junio, 2003. 
Hemos tenido la idea de que si le cambiamos el nombre a nuestras licenciaturas y posgrados tendremos más vigencia y hemos dejado de lado que el nombre conlleva un sinfín de significados y cargas paradigmáticas, lo cual no sólo implica seguir una corriente nominal "moderna" sino que es toda una nueva manera de concebir a la disciplina.

Mención aparte merece considerar que también hacemos documentación, porque de acuerdo con una aproximación somera a esta disciplina su objeto de estudio es el documento, no la información, no la relación información-usuario; es decir, la brecha paradigmática se agranda aún más.

Tenemos pues que establecer los límites de nuestras disciplinas, sus objetos de estudio y sus maneras de actuar.

Otro elemento que conforma un paradigma es la metodología, en este momento podemos preguntar, ¿qué metodología o metodologías se usan en bibliotecología?

Para responder esta pregunta hice un análisis que quedó registrado en el artículo Análisis metodológico para abordar el fenómeno usuarios de la información en América Latina, trabajo que aborda los métodos y técnicas que se han utilizado para desarrollar investigaciones. Cito a continuación algunas de las conclusiones:

[...] las tendencias actuales para abordar fenómenos bibliotecológicos... apuntan hacia corrientes y métodos clásicos asociados a las ciencias naturales, como son el método científico, el positivismo y la Teoría General de Sistemas, [...] algunos principios del método inductivo y, en menor medida, el deductivo y el analítico/ sintético.

La precisión conceptual de tales corrientes y métodos nos demuestra que, en realidad, sólo se siguen algunos principios y que falta claridad en la aplicación de ellos. [...] la tendencia de retomar métodos de otras disciplinas sin reflexionar críticamente si son los más convenientes. ${ }^{25}$

Con la información que hemos presentado acerca del objeto de estudio y las metodologías usadas estaremos en condiciones de esbozar el paradigma de la bibliotecología: es una disciplina cuyo objeto de estudio es la relación entre información y usuario, y cuyo interés básico se centra en la organización y sigue el método científico, el cual está apoyado en métodos cuantitativos; nuestra comunidad epistémica no está bien consolidada por la falta de intercambio, aplicación y replicación de resultados.

25 Patricia Hernández Salazar, "Análisis metodológico para abordar el fenómeno usuarios de la información en América Latina”, en Investigación Bibliotecológica: archivonomía, bibliotecología e información. 17 (35): 29. Julio/diciembre, 2003. 


\section{CONCLUSIONES Y SUGERENCIAS}

La investigación bibliotecológica en América Latina tiene varias décadas de haberse iniciado (entre dos y cuatro décadas) y sin embargo todavía siguen existiendo algunos retos que debemos enfrentar, como son la generación de marcos teóricos y la formación metodológica de los investigadores, retos que aparecen desde el origen del proceso, y que siguen vigentes.

En cuanto a la generación de marcos teóricos resalta el hecho de que algunos países (Argentina y Colombia) no han podido establecer sus objetos de estudio, o declararlos formalmente. La delimitación de los fenómenos, los campos y los subcampos se ha dado de acuerdo con los problemas que han ido surgiendo.

Esta generación está en franca relación con la determinación de los paradigmas que rigen las disciplinas, por lo que es preciso delimitar con claridad este paradigma, declararlo, y sobre esa base construir nuestro andamiaje teórico.

El desarrollo de una disciplina está en relación con los cuadros de investigadores que la crean, entre mayor sea su capacidad investigativa mayor será su desarrollo. Una de las formas de aumentar la capacidad de investigación es la obtención de niveles académicos de posgrado, concretamente el doctorado. Se hace pues necesario seguir avanzando en nuestra formación académica y adquirir mayor experiencia en el proceso de producción de conocimiento auténtico.

Otra alternativa es que las instituciones de educación que imparten los programas de bibliotecología y disciplinas afines, refuercen o empiecen a seguir la tendencia de formar para investigar desde los niveles de licenciatura.

Un compromiso que no se ha cubierto del todo es la producción de recursos que apoyen el desarrollo de nuestras disciplinas. Es conveniente registrar nuestros hallazgos y ponerlos a consideración de todos los interesados, además de crear los mecanismos necesarios para lograr una adecuada distribución.

Para evitar el trabajo aislado y sin reconocimiento de nuestros pares tendremos que organizar una mayor cantidad de eventos regionales de investigación, cuyo fin sea exponer y discutir los resultados que se han encontrado, y lograr conclusiones compartidas y aceptadas, es decir, validadas por una comunidad.

Lo que nos lleva a declarar que debemos construir una comunidad epistémica que se apoye, que esté enterada de los avances, que los difunda, que los aplique, replique, discuta y mejore, sólo así tendremos la certeza de no estar trabajando como entes aislados. 


\section{OBRAS CONSULTADAS}

Bachelard, G. La formación del espíritu científico. México: Siglo XXI, $1980.302 \mathrm{p}$.

Centro Universitario de Investigaciones Bibliotecológicas. México: UNAM, Centro Universitario de Investigaciones Bibliotecológicas, [s.f.]. $18 \mathrm{p}$.

Coloquio de Investigación Bibliotecológica y de la Información $\left(21^{\circ}\right.$ : 2003: México, D.F.). La investigación bibliotecológica en la era de la información: memoria del XXI Coloquio de Investigación Bibliotecológica y de la Información, 24-26 de septiembre de 2003. México: UNAM, Centro Universitario de Investigaciones Bibliotecológicas, 2004. 317 p.

Figueiredo, Nice. "Research in library and information sciences in Brazil", en Seminario pre-conferencia IFLA sobre educación para la investigación e investigación para la educación. Bogotá: ALEBCI, 1983. pp. 72-78.

Hernández Salazar, Patricia. "Análisis metodológico para abordar el fenómeno usuarios de la información en América Latina”, en Investigación Bibliotecológica: archivonomía, bibliotecología e información 17 (35): 5-31. Julio/diciembre, 2003.

Herrera Cortés, Rocío. "Panorama de educación e investigación de la Bibliotecología y Ciencias de la Información en Colombia”, en Ramírez Leyva, Elsa, Reunión de Investigadores y Educadores de Iberoamérica y del Caribe en el Área de Bibliotecología y Ciencia de la Información. México: UNAM, Centro Universitario de Investigaciones Bibliotecológicas, 1996. Pp. 248-264.

Informe de actividades agosto 2003-agosto 2004. México: Universidad Nacional Autónoma de México, 2004. 85 p.

Kuhn, Thomas S. The structure of scientific revolutions. Madrid: Tecnos, 1962. 210p.

Legislación universitaria de la UNAM. México: UNAM, 2000. 623 p.

Molina Escobar, María Clemencia. "Desarrollo y perspectiva de la investigación en bibliotecología y archivología en Colombia”, en Encuentro de Educadores e Investigadores de Bibliotecología, Archivología y Ciencia de la Información de Iberoamérica y el Caribe (4:1998: Maracaibo, Venezuela). Venezuela: [s.e.], 1998. 17 h.

Morales Campos, Estela. "Investigación Bibliotecológica", en Jornadas Mexicanas de Biblioteconomía (16: 1985: Pachuca, Hgo.). Memorias. México: AMBAC, 1986. pp. 21-30.

, Las posibilidades de la investigación bibliotecológica en América Latina. Boletín de la Asociación Andaluza de Bibliotecarios. 4 (10): 21-24. Enero-marzo, 1988.

Mueller, Suzana P. M., Antonio L.C. Miranda y Emir J. Suaiden. A pesquisa em Ciencia da informacao no Brasil: analise dos trábalhos apresentados no IV Enancib, Brasilia: 2000. Documento impreso en computadora. [17 p.]. 
Naranjo Vélez, Edilma y Nora Elena Rendón Giraldo. "La investigación bibliotecológica y las comunidades científicas en un contexto colombiano", en La investigación bibliotecológica en la era de la información: Memoria del XXI Coloquio de Investigación Bibliotecológica y de la Información, 24-26 de septiembre de 2003. México: UNAM, Centro Universitario de Investigaciones Bibliotecológicas, 2004. pp. 77-91.

Oliveira, Marlene de. "La investigación científica en ciencia de la información: análisis de la investigación financiada por el CNPQ (Brasil) en el período de 1984 a 1993”, en Investigación bibliotecológica: archivonomía, bibliotecología e información. 17 (34) : p. 70-87. Enero-junio, 2003.

Ortega y Gasset, José. Origen y epílogo de la filosofía. México: Fondo de Cultura Económica, c1998. 113 p.

Rodríguez Gallardo, Adolfo. "La investigación bibliotecológica”, en Jornadas Mexicanas de Biblioteconomia (8: 1977: Guadalajara, Jal.). La problemática de las bibliotecas en México y sus soluciones: memorias. México : AMBAC, 1977. pp. 29-38.

Romanos de Tiratel, Susana. "La investigación bibliotecológica en Argentina", en La investigación bibliotecológica en la era de la información: Memoria del XXI Coloquio de Investigación Bibliotecológica y de la Información, 24-26 de septiembre de 2003. México: UNAM, Centro Universitario de Investigaciones Bibliotecológicas, 2004. pp. 92-119.

Sabor, Josefa E. La investigación en bibliotecología. Buenos Aires: Asociación de Bibliotecarios Graduados de la República de Argentina, 1985.10 p.

\section{CUADROS}

Cuadro 1: Temas de investigación en la década de los ochenta

\begin{tabular}{|c|c|c|c|c|}
\hline Temas & $\begin{array}{l}\text { Argentina } \\
1985\end{array}$ & $\begin{array}{l}\text { México } \\
1985\end{array}$ & $\begin{array}{c}\text { Colombia } \\
1985\end{array}$ & $\begin{array}{c}\text { Brasil } \\
1978-1983\end{array}$ \\
\hline 1. Bibliografía nacional/bases & $X$ & & & \\
\hline 2. Servicios bibliotecarios & $X$ & & & \\
\hline 3.Procesos técnicos/análisis bibliográfico & $X$ & $X$ & & \\
\hline 4. Historia de la bibliotecología & & $X$ & $X$ & \\
\hline 5. Educación & & $X$ & $x$ & $X$ \\
\hline 6. Usuarios: estudios & & $x$ & $X$ & $x$ \\
\hline $\begin{array}{l}\text { 7. Evaluación: colecciones, servicios, unidades, } \\
\text { diseño, operatividad }\end{array}$ & & $x$ & $x$ & $x$ \\
\hline 8. Automatización & & $X$ & & \\
\hline 9. Selección & & $x$ & & \\
\hline 10. Tipos de biblioteca & & $X$ & & \\
\hline $\begin{array}{l}\text { 11.Teoría y estructura de la información } \\
\text { (bibliometría y cienciometría) }\end{array}$ & & & $x$ & \\
\hline $\begin{array}{l}\text { 12. Epistemología de la bibliotecología y de la } \\
\text { ciencia de la información }\end{array}$ & & & $x$ & \\
\hline 13. Promoción de la lectura & & & $x$ & \\
\hline
\end{tabular}




\begin{tabular}{|c|c|c|c|c|c|}
\hline 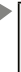 & Temas & $\begin{array}{c}\text { Argentina } \\
1985\end{array}$ & $\begin{array}{c}\text { México } \\
1985\end{array}$ & $\begin{array}{c}\text { Colombia } \\
1985\end{array}$ & $\begin{array}{c}\text { Brasil } \\
1978-1983\end{array}$ \\
\hline & 14. Lenguajes de indización & & & & $x$ \\
\hline & 15. Sistemas bibliotecarios & & & & $x$ \\
\hline & 16. Transferencia de información & & & & $x$ \\
\hline & 17. Bibliotecas públicas & & & & $x$ \\
\hline
\end{tabular}

Cuadro 2: Problemas en la investigación bibliotecológica : antecedentes

\begin{tabular}{|c|c|c|c|c|}
\hline Problemas & $\begin{array}{c}\text { Argentina } \\
1985\end{array}$ & $\begin{array}{c}\text { México } \\
1985\end{array}$ & $\begin{array}{c}\text { Colombia } \\
1993\end{array}$ & $\begin{array}{c}\text { Brasil } \\
1978-1983\end{array}$ \\
\hline 1. Falta de principios teóricos & $x$ & & $x$ & $x$ \\
\hline $\begin{array}{l}\text { 2. Poco interés por cuestionar principios } \\
\text { aceptados }\end{array}$ & $x$ & & & \\
\hline 3. Inexistencia de metodología & $x$ & & & $x$ \\
\hline $\begin{array}{l}\text { 4. Falta de recursos con aptitudes } \\
\text { y actitudes para investigar }\end{array}$ & $x$ & $x$ & $x$ & $x$ \\
\hline 5. Salarios bajos & & $x$ & & \\
\hline $\begin{array}{l}\text { 6. Presupuesto: carencia para formarse y } \\
\text { actualizarse; recortes, inadecuado }\end{array}$ & & $x$ & & $x$ \\
\hline $\begin{array}{l}\text { 7. Infraestructura deficiente: equipos, } \\
\text { colecciones, instalaciones }\end{array}$ & & $x$ & & $x$ \\
\hline $\begin{array}{l}\text { 8. Mecanismos deficientes de difusión de } \\
\text { resultados }\end{array}$ & & $x$ & $x$ & \\
\hline $\begin{array}{l}\text { 9. Elevado número de investigaciones } \\
\text { exploratorio-descriptivas }\end{array}$ & & & $x$ & \\
\hline 10. No existe comprobación de tesis & & & $x$ & \\
\hline $\begin{array}{l}\text { 11. Aislamiento de investigadores: } \\
\text { nivel nacional y regional }\end{array}$ & & & $x$ & $x$ \\
\hline $\begin{array}{l}\text { 12. Ausencia de retroalimentación: réplica de } \\
\text { investigaciones, confrontación de resultados }\end{array}$ & & & $x$ & \\
\hline 13. Escasa producción & & & $x$ & \\
\hline $\begin{array}{l}\text { 14. Inexistencia de grupos dedicados } \\
\text { exclusivamente a la investigación }\end{array}$ & & & $x$ & $x$ \\
\hline 15. No existe tradición en investigación & & & & $x$ \\
\hline $\begin{array}{l}\text { 16. Comunicación deficiente en los colegios } \\
\text { invisibles }\end{array}$ & & & & $x$ \\
\hline
\end{tabular}

Cuadro 3: Soluciones a problemas : antecedentes

\begin{tabular}{|l|c|c|c|c|}
\hline \multicolumn{1}{|c|}{ Temas } & $\begin{array}{c}\text { Argentina } \\
1985\end{array}$ & $\begin{array}{c}\text { México } \\
1985\end{array}$ & $\begin{array}{c}\text { Colombia } \\
1993\end{array}$ & $\begin{array}{c}\text { Brasil } \\
1978-1983\end{array}$ \\
\hline $\begin{array}{l}\text { 1. Formación eficaz sobre metodología de la } \\
\text { investigación por parte de las escuelas }\end{array}$ & $X$ & & & \\
\hline $\begin{array}{l}\text { 2. Motivar a los docentes a realizar trabajos de } \\
\text { investigación }\end{array}$ & $X$ & & & \\
\hline 3. Formular planes de trabajo definidos & $X$ & & & \\
\hline
\end{tabular}




\begin{tabular}{|c|c|c|c|c|}
\hline Temas & $\begin{array}{c}\text { Argentina } \\
1985\end{array}$ & $\begin{array}{c}\text { México } \\
1985\end{array}$ & $\begin{array}{c}\text { Colombia } \\
1993\end{array}$ & $\begin{array}{c}\text { Brasil } \\
1978-1983\end{array}$ \\
\hline $\begin{array}{l}\text { 4. Conformar colecciones sobre bibliotecología } \\
\text { latinoamericana }\end{array}$ & & $x$ & & \\
\hline $\begin{array}{l}\text { 5. Crear bibliotecas especializadas en } \\
\text { bibliotecología }\end{array}$ & & $x$ & & \\
\hline $\begin{array}{l}\text { 6. Formar al personal en metodología de la } \\
\text { investigación, estadística, automatización y } \\
\text { tecnología educativa }\end{array}$ & & $x$ & $x$ & $x$ \\
\hline 7. Desarrollar proyectos colectivos & & & $x$ & \\
\hline 8. Difundir los resultados & & & $x$ & \\
\hline 9. Trabajar entre pares & & & $x$ & \\
\hline 10. Crear la formación de investigadores & & & $\mathrm{X}$ & \\
\hline 11. Capacitar administradores de investigación & & & $x$ & \\
\hline 12. Retomar teorías de otras disciplinas & & & & $x$ \\
\hline 13. Utilizar métodos adecuados & & & & $x$ \\
\hline
\end{tabular}

Cuadro 4: Temas de investigación en la década del 2000

\begin{tabular}{|l|c|c|c|c|}
\hline \multicolumn{1}{|c|}{ Temas } & $\begin{array}{c}\text { Argentina } \\
\mathbf{2 0 0 3}\end{array}$ & $\begin{array}{c}\text { México } \\
\mathbf{2 0 0 4}\end{array}$ & $\begin{array}{c}\text { Colombia } \\
\mathbf{2 0 0 3}\end{array}$ & $\begin{array}{c}\text { Brasil } \\
2000\end{array}$ \\
\hline 1. Búsqueda: procesos, lenguaje & $\mathrm{X}$ & $\mathrm{X}$ & & \\
\hline $\begin{array}{l}\text { 2. Automatización: bibliotecas universitarias, } \\
\text { impacto }\end{array}$ & 2 & & & \\
\hline $\begin{array}{l}\text { 3. Indicadores: actividad de investigación, } \\
\text { producción en economía, historia y sociología }\end{array}$ & 2 & & & \\
\hline $\begin{array}{l}\text { 4. Historia: libro, bibliotecas, lectura, } \\
\text { bibliotecología }\end{array}$ & $\mathrm{X}$ & $\mathrm{X}$ & & \\
\hline 5. Bibliotecas públicas & $\mathrm{X}$ & & & \\
\hline $\begin{array}{l}\text { 6. Control bibliográfico: en ciencias } \\
\text { humanas, normalización }\end{array}$ & $\mathrm{X}$ & $\mathrm{X}$ & & \\
\hline $\begin{array}{l}\text { 7. Fundamentación : epistemológica, ética, } \\
\text { histórico-social }\end{array}$ & & 3 & & $X$ \\
\hline $\begin{array}{l}\text { 8. Educación: formación profesional / mercado } \\
\text { de trabajo }\end{array}$ & & $X$ & & $X$ \\
\hline 9. Lectura & & $X$ & & \\
\hline 10. Políticas y legislación de la información & & $X$ & & \\
\hline 11. Desarrollo de colecciones & & $X$ & & \\
\hline 12. Metría de la información & & $X$ & & \\
\hline 13. Fuentes de información & & $X$ & & \\
\hline $\begin{array}{l}\text { 14. Usuarios de la información: estudios, } \\
\text { información y sociedad }\end{array}$ & & 2 & & $X$ \\
\hline 15. Sistemas de clasificación & & & & \\
\hline $\begin{array}{l}\text { 16. Tecnologías de información: marco } \\
\text { conceptual, evolución, recursos de información, } \\
\text { interfases para transferencia y acceso a la } \\
\text { información digital }\end{array}$ & & & & \\
\hline 17. Contextualización de la información & & & \\
\hline
\end{tabular}




\begin{tabular}{|c|c|c|c|c|c|}
\hline 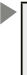 & Temas & $\begin{array}{c}\text { Argentina } \\
2003\end{array}$ & $\begin{array}{l}\text { México } \\
2004\end{array}$ & $\begin{array}{c}\text { Colombia } \\
2003\end{array}$ & $\begin{array}{l}\text { Brasil } \\
2000\end{array}$ \\
\hline & $\begin{array}{l}\text { 18. Sistemas de información: caracterización, } \\
\text { teoría, planeación/inteligencia competitiva, } \\
\text { gerencia }\end{array}$ & & & $x$ & $x$ \\
\hline & 19. Organización & & & $x$ & \\
\hline & 20. Educación a distancia y tecnologías & & & & $x$ \\
\hline & 21. Información tecnológica & & & & $x$ \\
\hline & 22. Comunicación científica & & & & $\mathrm{x}$ \\
\hline & 23. Representación del conocimiento/indización & & & & $x$ \\
\hline
\end{tabular}

Cuadro 5. Problemas en la investigación bibliotecológica : década del 2000

\begin{tabular}{|l|c|c|c|c|}
\hline \multicolumn{1}{|c|}{ Temas } & $\begin{array}{c}\text { Argentina } \\
2003\end{array}$ & $\begin{array}{c}\text { México } \\
\mathbf{2 0 0 4}\end{array}$ & $\begin{array}{c}\text { Colombia } \\
2003\end{array}$ & $\begin{array}{c}\text { Brasil } \\
2000\end{array}$ \\
\hline 1. Preeminencia de la investigación aplicada & $X$ & & & \\
\hline 2. Ausencia de investigaciones teóricas & $X$ & & & \\
\hline $\begin{array}{l}\text { 3. Carencia de conocimientos sobre métodos y } \\
\text { técnicas para investigar y para recolectar datos }\end{array}$ & $X$ & & & $X$ \\
\hline 4. Construcción empírica de conocimientos & $X$ & & & \\
\hline $\begin{array}{l}\text { 5. Barreras de comunicación entre profesionales } \\
\text { e investigadores }\end{array}$ & $X$ & & & $X$ \\
\hline $\begin{array}{l}\text { 6. Falta de aplicación de los resultados de } \\
\text { investigación por parte de los profesionales }\end{array}$ & $X$ & $X$ & & \\
\hline $\begin{array}{l}\text { 7. Mecanismos de difusión deficientes 0 } \\
\text { inexistentes }\end{array}$ & $X$ & $X$ & & \\
\hline 8. Baja producción de literatura bibliotecológica & $X$ & $X$ & & \\
\hline 9. Falta de formación para investigar & $X$ & $X$ & & \\
\hline 10. Uso indiscriminado de métodos cuantitativos & & $X$ & $X$ & \\
\hline 11. Trabajo individual & & $X$ & & \\
\hline 12. Falta de replicabilidad & & $X$ & & \\
\hline $\begin{array}{l}\text { 13. Escasa generación de marcos teóricos y } \\
\text { conocimiento auténtico }\end{array}$ & & $X$ & $X$ & \\
\hline 14. Carencia de un campo teórico sólido & & & $X$ & \\
\hline $\begin{array}{l}\text { 15. No hay aceptación de marcos teóricos por } \\
\text { comunidades epistémicas }\end{array}$ & & & $X$ & \\
\hline 16. Falta de tradición en investigación & & & $X$ & \\
\hline $\begin{array}{l}\text { 17. No se tiene conciencia de la importancia de } \\
\text { la investigación para el desarrollo de la profesión }\end{array}$ & & & $X$ & \\
\hline $\begin{array}{l}\text { 18. Deficiencia en el uso de métodos y técnicas } \\
\text { de investigación }\end{array}$ & & & & \\
\hline $\begin{array}{l}\text { 19. La mayoría de trabajos son producto de } \\
\text { estudiantes de maestría }\end{array}$ & & & & \\
\hline
\end{tabular}

